\title{
4 Sinnherstellung in der Organisation Schule
}

Orientierungen der Förderung und Selektion sowie Bildungsgerechtigkeitskonzeptionen sind weder bei einzelnen Akteuren noch in schulischen Kollektiven einfach gegeben, sondern können als Ergebnis gesellschaftlicher und politischer Debatten, der Sozialisation sowie der Kommunikation und Reflexion innerhalb der jeweiligen Organisation angesehen werden. In Schulen sind verschiedene Akteure und Professionen vertreten, die in ihrer Interaktion das Handlungswissen der einzelnen Akteure auch gerade mit Blick auf Förderung und Selektion mitprägen. Auch die Organisation selbst kann aktiv oder passiv dazu beitragen, kollektives Handlungswissen zu generieren. Eine mehrperspektivische Sichtweise geht davon aus, dass die Organisation nicht nur die Summe ihrer Akteure und Akteursgruppen darstellt, sondern auch darüber hinaus eine eigene Wirkung entfaltet. Damit stellt sich die Frage, welchen Zusammenhang die Organisation als handlungsleitender Erfahrungsraum mit den unterschiedlichen Bildungsgerechtigkeitskonzeptionen aufweist und wie sich dies empirisch überprüfen lässt. Hierzu ist es erforderlich, sich zunächst Klarheit über theoretische Konzepte und empirische Befunde zur Sinnherstellung in der Organisation Schule zu verschaffen. Dem Begriff der Sinnherstellung liegt ein konstruktivistisches Verständnis im Sinne von Schütz (1971) zugrunde. Dieses Verständnis beruht auf der Annahme, dass Wissen, sei es im wissenschaftlichen oder im alltäglichen Bereich, Konstruktionsprozessen unterliegt. Kollektive Orientierungen der Förderung und Selektion und der Bildungsgerechtigkeit unterliegen einem aktiven Herstellungsprozess, den es zu ergründen gilt. Auf der Ebene der pädagogischen Akteure ist die Schule als Organisation aufgrund der Inklusionsbestrebungen und der Einführung geleiteter Schulen eine zunehmend multiprofessionelle Organisation. Es lohnt sich, einen Blick zu werfen auf den unterschiedlichen Habitus der einzelnen Professionen und auf die Passungsverhältnisse zwischen den Berufsgruppen, aber auch in ihrem Bezug zur vorhandenen Klientel. Daneben kann zudem die Schule selbst als Akteur gesehen werden, der zur Herstellung gemeinsamer Vorstellungen und Konzeptionen beiträgt. Das Schulsystem und die Organisation können einerseits aufgrund der ihnen eingeschriebenen Bedingungen mit Bildungsgerechtigkeitsvorstellungen in Konflikt geraten, was in einer systemtheoretischen Betrachtung verschiedener Funktionen schulischer Organisationen zu analysieren ist. Andererseits kann die Organisation als Akteur zur Bearbeitung dieser Konflikte beitragen, wobei Aushandlungsprozesse und Interdependenzen verschiedener Kontexturen mitzudenken sind. Abschließend wird das Konzept der Schulkultur reflektiert, das als ,Endprodukt ${ }^{6}$ eines Sinnherstellungsprozesses angesehen werden kann. Diese theoretischen und empirischen Reflexionen dienen dazu, ergänzend zu einer akteurs- oder 
professionszentrierten Perspektive den Fokus auf die Organisation als kollektives Konstrukt zu richten, welches für die Herstellung von Sinn von Bedeutung sein kann.

\subsection{Professioneller Habitus und Passungsverhältnisse}

Studien, die sich mit dem jeweiligen Habitus von Professionen und den sich daraus ergebenden Passungsverhältnissen auseinandersetzen, beziehen sich auf das Konzept des Habitus, welches Pierre Bourdieu (1982) insbesondere in seinem Werk Die feinen Unterschiede ausdifferenziert hat. Der Habitus wird als unbewusstes Wahrnehmungs-, Deutungs- und Handlungsschema verstanden, das sich aus der jeweiligen Position in der Gesellschaft ergibt (Bourdieu, 1982). Im Kontext der Schule als Bildungseinrichtung wird auf der einen Seite der Frage nachgegangen, inwiefern unbewusste Passungsproblematiken zwischen dem Habitus schulischer Akteure und demjenigen ihres sozialen Kontextes pädagogisches Handeln beeinflussen. In der Frage nach Passungsverhältnissen zur Lebenswelt der Schüler/-innen und ihrer Familien wird auf die theoretischen und empirischen Überlegungen zur Mittelstandsorientierung des Erziehungssystems nach Bourdieu und Passeron (1971) Bezug genommen. Im Zentrum der Untersuchungen stehen unbewusste Passungsproblematiken zwischen Lehrpersonen und Schüler/-innen respektive deren Eltern. Für die vorliegende Arbeit sind diese Studien insofern relevant, als aufgezeigt wird, inwiefern unbewusste Sinnstrukturen in der Bearbeitung von Chancengerechtigkeit in der Organisation der Förder- und Selektionsaufgabe mitspielen können. Während in dieser ersten Perspektive der Habitus als $\mathrm{Zu}$ gehörigkeit von Akteuren zu einer Position im sozialen Raum verstanden wird, kann auf der anderen Seite gefragt werden, welche unbewussten Deutungsschemata eines professionellen Habitus das Handeln der Akteure leitet. In dieser Perspektive wird das Konstrukt der Klassenzugehörigkeit auf die Professionszugehörigkeit übertragen und untersucht, inwiefern unbewusste Deutungs- und Handlungsschemata einer professionellen Zugehörigkeit zugeschrieben sein können. Dabei werden einerseits einzelne pädagogische Professionen, andererseits auch Professionskonstellationen untersucht. Die zweite Perspektive schneidet somit die Forschungsdiskussion zu multiprofessionellen Teams an, die aber in der vorliegenden Arbeit nicht im Zentrum stehen.

Studien zu Passungsverhältnissen untersuchen insbesondere die mehr oder weniger große Nähe der milieuspezifischen Eigenschaften der Schüler/-innen respektive ihrer Eltern im Vergleich zu den Schulakteuren bzw. der Schule (vgl. u. a. Bourdieu \& Passeron, 1971; Kramer \& Helsper, 2010). Da der Bildungshabitus ein latentes Konstrukt darstellt, wird diesem Passungsverhältnis mehrheitlich in rekonstruktiven Studien nachgegangen. Kramer und Helsper (2010) zeigen auf der Basis einer Interviewstudie mit Schülerinnen und Schülern, dass das Erleben insbesondere des Typus „Habitus der Bildungsfremdheit“ mit Blick auf schulische Anforderungen mit einem drohenden chronischen Misserfolg zusammenhängt. Dabei erweist sich im Vergleich 
verschiedener Schulformen das schulkulturelle Institutionenmilieu als ausschlaggebend dafür, dass Anschlusslösungen von Schülerinnen und Schülern aus nahen Milieus eröffnet und von solchen aus fernen Milieus geschlossen werden (ebd.). Helsper, Thiersch und Ziems (2013) untersuchen zudem die Veränderung des Bildungshabitus der Schüler/-innen im Laufe ihrer Schulkarriere. Diese hängt den Autoren zufolge eng mit den Praktiken und Strukturen der sozialen Welt, insbesondere der Institutionen des Bildungssystems, zusammen.

Mit Blick auf den Habitus von Lehrpersonen untersucht Amling (2015) auf der Basis von Gruppendiskussionen unterschiedliche Lernorientierungen von Grundschullehrerinnen und -lehrern. Dabei versucht er, die Reproduktion sozialer Ungleichheit relational zu erfassen, indem der jeweilige milieuspezifische Habitus den Regeln und Sinnstrukturen des schulischen Feldes gegenübergestellt wird (Amling, 2015). Im Ergebnis lässt sich die Bedeutung der Schule als Organisation für die Herausbildung dieser Lernorientierungen hervorheben (Amling, 2015). Gemäß Bremm et al. (2016) ist die Passungsdifferenz zwischen Schüler/-innenhabitus und Akteuren auf Organisationsebene in sozial benachteiligte Bildungsräume besonders relevant, was zu fehlender Anschlussfähigkeit an geforderte Verhaltensweisen führen kann. Diese Differenz wird gemäß Fölker und Hertel (2015) von den pädagogischen Akteuren in ihrer Bezugnahme auf Lernende zumindest mitkonstruiert. Diese Bezüge betreffen verschiedene Akteure und Kontexte. Die Frage nach Passungsverhältnissen wird aus Sicht des Schüler/-innenhabitus, aus Sicht der Lehrpersonen und in der Diskrepanz dieser beiden Gruppen sowie mit Blick auf die Regeln der Bildungsorganisationen diskutiert.

Insgesamt findet sich für die von Bourdieu und Passeron (1971) im französischen (Gymnasiums-)Kontext entwickelte Theorie in der Forschung noch immer empirische Evidenz. Dabei untersuchen die Studien jedoch nicht nur milieuspezifische Merkmale von Individuen, sondern ebenfalls die Organisation (z. B. Amling, 2015) oder den soziostrukturellen Kontext (z. B. Bremm, 2016). Kritisch eingewendet wird allerdings, dass Konstruktionsleistungen der gängigen Kategorie der Bildungsferne und ihre meist unreflektierte Verwendung als (sozial-)pädagogische Normalitätsvorstellung pädagogischen Handelns zu überdenken sei (u. a. Wiezorek, 2009). Wiezorek (2009) sieht in der Kategorisierung der Bildungsferne von Schülerinnen und Schülern bzw. deren Eltern durch wissenschaftliche Studien und Schulakteure eine ungleichheitsreproduzierende Wirkmächtigkeit. Der Frage nach professionellen Handlungshintergründen wird in empirischen und theoretischen Studien ebenfalls ergebnisoffen begegnet. Im Anschluss geht es um die Analyse des professionellen Habitus verschiedener Akteure an schulischen Organisationen sowie ihres Zusammenspiels.

Die Lehrperson ist an schulischen Organisationen und im direkten Kontakt mit den Schülerinnen und Schülern der zentrale Akteur. Die Profession der Lehrperson wird in theoretischen und empirischen Konzepten oft in einem Spannungsverhältnis zu den institutionellen und organisationalen Ansprüchen beschrieben. Das theoretische Konstrukt des „Autonomie-Paritäts-Paradigmas“ (Lortie, 1975) beschreibt die hohe 
operative Autonomie der Lehrperson bei widersprüchlichen institutionellen Anforderungen an die Profession. In diesem Spannungsverhältnis sieht Terhart (2011) die Beibehaltung didaktischer Routinen als zentralen Bestandteil der Lehrpersonenprofession. Dabei zeigen sich in der unterrichtlichen Praxis Resistenzen gegenüber extern entwickelten Konzepten und Reformen, wobei die Lehrpersonen zwischen den Positionen Einzelkämpfer und Standardisierung stehen und unterschiedliche Deutungen von Reformen entwickeln (Terhart, 2011, S. 214). In Bezug auf die Schüler/-innen versucht der Ansatz struktureller Professionsantinomien die widersprüchlichen Ansprüche an Lehrpersonen als praktische Dilemmata zu fassen. Dabei werden pädagogische Orientierungen in der Antinomie „universalistisch-distanziert, rollenförmig“ versus „diffusnah, personenbezogen" gesehen (Helsper, 2011, S. 223). Auch bei den individuellpersonenbezogenen pädagogischen Orientierungen werden Spannungsverhältnisse reflektiert, die aus der zunehmend auf Leistungs- und Anstrengungsbereitschaft ausgerichteten Erwartungshaltung resultieren (Heinrich, 2010, S. 129).

Neben dem Antinomie-Ansatz gibt es zum professionsspezifischen Lehrpersonenhabitus Konzepte und Studien, die auf Orientierungen, subjektive Theorien und Überzeugungen abzielen (Reusser \& Pauli, 2014). Vorgeschlagen wird hier der Überbegriff der „berufsbezogenen Überzeugung“ für solche Facetten des Handelns von Lehrpersonen, welche über das pädagogische und fachliche Wissen hinausgehen (ebd., S. 642). Darunter wird in Anlehnung an das Habitus-Konzept nach Bourdieu (1974) ein berufsbiographisch verinnerlichtes unbewusstes Wissen verstanden, das bereits durch die eigene Schulzeit und Ausbildung, aber auch durch die Einbindung in Institutionen geprägt wird (Reusser \& Pauli, 2014). In der Erforschung berufsbezogener Überzeugungen überwiegen rekonstruktive Verfahren, wobei zunehmend auch quantitative Methoden eingesetzt werden, um die Zusammenhänge zwischen Überzeugungen und Unterrichtshandeln zu erkunden (ebd.). Unter dem Überbegriff der berufsbezogenen Überzeugungen werden sowohl kognitive Konzepte und pädagogische Vorstellungen guten Unterrichtens als auch „kontextbezogene Überzeugungen“ gefasst, was unter anderem Bildungsgerechtigkeitskonzeptionen von Lehrpersonen betrifft (ebd., S. 651). Verschiedene Studien untersuchen in dieser Forschungsperspektive Überzeugungen im Hinblick auf integrative Unterrichtsformen. Quantitative Arbeiten widmen sich dabei beispielsweise der Entstehung von Differenzierungen durch Lehrpersonen (bspw. Gebhardt et al., 2011) oder den Auswirkungen kontextbezogener Überzeugungen auf das Gelingen von Reformen (bspw. Beckstein, Reusser, Stebler \& Mandel, 2013).

Die durch ihre eigene Bildungsbiographie geprägten Lehrpersonen stehen in einem ständigen Aushandlungsprozess zwischen eigenen Gestaltungsanteilen und den unterschiedlichen Anforderungen der umgebenden Systeme. So zeichnet sich der Lehrpersonenhabitus durch sein In-Beziehung-Setzen zu institutionellen, sozialen und innerorganisationalen Anforderungen aus. Fend (2008) zufolge ist das Handeln der Schulakteure auf die strukturelle und soziale Umwelt gerichtet, da Erwartungen nicht 
nur schulintern entstehen, sondern auch system- oder kontextinduziert sind. Um Rahmenvorgaben und spezifische Handlungsbedingungen im Schulalltag berücksichtigen zu können, sind Rekontextualisierungsleistungen der Akteure auf den verschiedenen Ebenen nötig. Hier zeigt sich also ein aktiver Gestaltungsanteil im Kontext sich ständig wandelnder Anforderungen und Bedingungen (Fend, 2008, S. 26). Die institutionellen Vorgaben werden auf operativer Ebene als veränderbar betrachtet; grundlegend für die Rekontextualisierungsleistungen der Schulakteure ist dabei das Zusammenspiel der nächsthöheren Ebene (Vorgaben), der darunterliegenden Ebene (Folgewirkung, Konsequenzen des Handelns) und der horizontalen Ebene (Handlungserfahrungen) (Fend, 2008, S. 38). Gemäß Fend (2008) wird der Autonomieanspruch der Lehrperson durch alltägliche Aushandlungsprozesse der pädagogischen Arbeit im Abgleich mit Vorgaben, Reformen und Handlungsroutinen relativiert.

Im Hinblick auf innerorganisationale Aushandlungsprozesse an Primarschulen wird die multiprofessionelle Zusammenarbeit in zunehmend inklusiven Schulsystemen diskutiert. Ohne an dieser Stelle näher auf die Auswirkungen dieser Zusammenarbeit auf den Unterricht und die Schüler/-innen eingehen zu können, lohnt sich ein kurzer Blick auf die professionshabituellen Differenzierungen. So sah bereits vor zwanzig Jahren Wocken (1996) die Gefahr, dass bei der Einführung integrativer Fördersysteme Förderlehrpersonen die zunehmende Sensibilisierung für alle Arten von Auffälligkeiten als Legitimationsstrategie für ihre Arbeit gebrauchen könnten. Durch den Verlust der räumlichen Abgrenzung der integrationspädagogischen Arbeit in inklusiven Fördersystemen könnte die Legitimation zunehmend durch Etikettierungen von Schülerinnen und Schülern ,im Kopf der Pädagogen“ erfolgen (ebd., S. 35-36). In der Literatur wird dieses Phänomen „Etikettierungs-Ressourcen-Dilemma“ genannt (Wocken, 1996). Konstruiert wird das Bild einer Förderlehrperson, welche sich stark durch ihre sonderpädagogische Arbeit in Abgrenzung von der Arbeit der allgemeinen Lehrperson identifiziert.

$\mathrm{Zu}$ dieser Abgrenzung verschiedener professioneller Paradigmen gibt es insbesondere rekonstruktive Ansätze, welche die Kooperation verschiedener Schulakteure in inklusiven Settings als Spannungsfeld beschreiben (u. a. Graf \& Graf, 2008; Schröck \& Rahm, 2009). So werden an Schulen unterschiedliche professionelle Milieus ausgemacht, welche sich gegenüber dem Organisationsmilieu positionieren müssen. Sturm (2012) kommt beispielsweise zu dem Ergebnis, dass sich der Lehrpersonenhabitus insbesondere durch die Ausrichtung an schulisch-institutionellen Vorgaben der Selektion auszeichnet und sich vom Förderparadigma der Heilpädagogen abgrenzt. Die Untersuchung von Pool Maag und Moser Opitz (2014) im Schweizer Kontext zeigt ebenfalls die unterschiedlichen Sichtweisen der Klassenlehrpersonen und der Förderlehrpersonen auf die optimale Förderung. Anhand von Interviews werden die soziale Integration und das Wohlbefinden der Lernenden als zentraler Wert von Förderlehrpersonen herausgearbeitet, während die Lehrpersonen den Fokus auf die Leistungsförderung im Klassenverband richten (Pool Maag \& Moser Opitz, 2014). 
Die Selbstthematisierung des professionellen Habitus von Sonderpädagoginnen und -pädagogen in inklusiven Schulen untersuchen Heinrich, Arndt und Werning (2014). Gemäß den Autoren grenzen Lehrpersonen ihre professionelle Identität von anderen schulischen Professionalitäten ab. In einer Gegenüberstellung von Leistungsprinzipien (als klassische Lehrpersonendomäne) und Förderprinzipien (als klassische Förderlehrpersonendomäne) im schulischen Kontext reflektieren die Autor/-innen professionelle Deutungsmuster der Sonderpädagoginnen und -pädagogen. Sie zeigen, dass die Förderlehrperson gerade auch das ,professionsfremde' Leistungsprinzip übernimmt, um handlungsfähig zu bleiben (Heinrich, Arndt \& Werning, 2014). Dies erklären sie mit dem Verlust der Zuständigkeit für starke Schüler/-innen und der drohenden Abwertung der Arbeit der Förderlehrperson durch die sonderpädagogische Expertise (ebd., S. 67). In der Forschung wird somit der professionelle Habitus von Förderlehrpersonen in der Abgrenzung von dem der Lehrpersonen definiert. Klassische $\mathrm{Zu}-$ schreibungen der Förderlehrperson mit Fokus auf das Kind und seine Bedürfnisse (Förderung) und der Lehrperson mit Fokus auf die Klasse und die Leistung der Schüler/-innen (Selektion) werden durch integrativen Unterricht entweder in Frage gestellt oder aufgrund der Suche nach Legitimität gerade verfestigt.

An den Primarschulen in der Schweiz gibt es flächendeckend seit der Volksschulreform von 2005 neben Lehrpersonen und verschiedenen Heilpädagogen als weitere Akteure die Schulleitung. Durch die als obligatorisch erklärte Ausbildung legitimiert sich seither die Schulleitung als eigene Profession (Graf \& Graf, 2008). Schulleitungen stellen im Schweizer Kontext somit eine relativ junge Profession dar, wobei in der Praxis beispielsweise der Verband der Schulleiter/-innen (VSL) versucht, durch ein Leitbild eine Profilierung, aber auch eine Abgrenzung von anderen Schulakteuren zu erreichen (Verband Schulleiterinnen und Schulleiter Schweiz, 2015). Die Schulleitung hat ihrem Berufsbild zufolge eine Triagefunktion zwischen den Ansprüchen von Lehrpersonen, Schülerinnen bzw. Schülern, Eltern und Behörden und ist für die Entwicklung, Qualitätskontrolle und operative Führung der Schule verantwortlich (Dubs, 2010). Zudem wird der Profession des Schulleiters eine primäre Verantwortung in der Organisationsentwicklung zugeschrieben. Internationale Forschungen bezeichnen Schulleitungen im Hinblick auf den Erfolg von Entwicklungsprozessen als ,driver for change" (Bryk, 2010). Für Fend (2008, S. 170) zeichnet sich, was die Schulentwicklung betrifft, eine ,gute Schulleitung“ durch Charakteristika wie „kompetent“, „lernfähig, offen und entwicklungsorientiert" sowie dadurch aus, dass sie das Kollegium in Entscheidungs- und Planungsprozesse einbezieht. Die Profession der Schulleitung ist gemäß diesen Studien von der ständigen Aushandlung zwischen Bedürfnissen verschiedener Akteure einerseits und Anforderungen an Entwicklungs- und Reformhandeln andererseits geprägt. Der professionelle Habitus der Schulleitung wird entsprechend in engem Zusammenhang mit organisationaler und personaler Weiterentwicklung gesehen. 
Diese theoretischen und empirischen Arbeiten geben Hinweise auf Unterschiede, potentielle Spannungen und Aushandlungen zwischen verschiedenen Berufsgruppen mit ihrem jeweiligen Habitus in der Organisation Schule. In den Studien zu Passungsverhältnissen wird Habitus in seiner klassischen Kontierung als unbewusstes Deutungs- und Handlungsschema, das auf der eigenen Positionierung im gesellschaftlichen Raum basiert, verstanden. In einer solchen Perspektive wird der Habitus der Schulakteure von dem der Schüler/-innen und ihrer Eltern abgegrenzt. Wird hingegen das Spannungsfeld zwischen verschiedenen schulischen Akteuren betrachtet, ist vielmehr ein auf der Bildungssozialisation beruhender Habitus gemeint. Dabei werden Deutungsmuster dem professionellen Handlungs- oder Kontextwissen zugeschrieben und voneinander abgegrenzt. Verschiedenen Studien, welche den unterschiedlichen professionellen Habitus und die jeweiligen Passungswahrnehmungen untersuchen, betonen den Einfluss der organisationalen und systemischen Eingebundenheit im schulischen Handeln. Diese Eingebundenheit dürfte auch dafür relevant sein, inwiefern die Organisation selbst handlungsleitend wirken kann. Der Bezug der Einzelakteure zu einer überindividuellen Struktur in Organisationen wird beispielsweise in der SensemakingTheorie reflektiert. Anknüpfend an den Rekontextualisierungsansatz nach Fend werden im Folgenden solche Sensemaking-Prozesse in den Blick genommen.

\subsection{Rekontextualisierung und Sensemaking in Bildungsorganisationen}

Das Konzept der Rekontextualisierung kann nach Fend (2008, S. 26) als Ausdifferenzierung des Modells von Input (Rahmenvorgaben), Prozess und Output verstanden werden. Die „verführerische“ Einfachheit der darin enthaltenen Abhängigkeitsbeziehung ist gemäß dem Autor zu spezifizieren (ebd., S. 26). Schulische Organisationen und Akteure sind keine isolierten Einheiten, sondern in ihren Kontext eingebunden. Rekontextualisierung bedeutet hier, dass bildungspolitische Rahmenbedingungen kontextspezifisch in der Selbst- und Fremdwahrnehmung adaptiert werden (ebd., S. 27). Das heißt, Rahmenbedingungen werden nicht einfach übernommen, sondern sind von den Akteuren aktiv mitgestaltet. Dies wirft die Frage auf, inwiefern der aktive Gestaltungsanteil der Schulakteure von organisationalen kollektiven Strukturen mitgeprägt ist.

Eine mögliche theoretische Antwort bietet das Sensemaking-Konzept. So betrachtet Weick (1976) schulische Organisationen als loosely coupled systems, die sich innerorganisatorisch nicht durch Intensität und Dichte, sondern eine Vielzahl loser Verbindungen auszeichnen. Die geringe Dichte ermöglicht ein dauerhaftes, überindividuelles Bestehen schulischer Organisationen trotz oder gerade aufgrund kontext- und akteursspezifischer Adaptionsmöglichkeiten (Weick, 1976, S. 6-7). Diese losen Verbindungen und akteursspezifischen Gestaltungsmöglichkeiten bedeuten gemäß der Theorie nicht, dass Handlungsroutinen völlig frei von kollektivem Sinn gestaltet sind. 
So sieht Weick (1976, S. 13) in lose gekoppelten Organisationen einen erhöhten Bedarf, gemeinsame Konzepte und Handlungsstrategien auszuhandeln. Das Konzept des Sensemakings in Organisationen kann als Antwort auf die Frage gesehen werden, wie innerhalb der Organisation Wertesysteme konstruiert und reformuliert werden (Weick, Sutcliffe \& Obstfeld, 2005). Sensemaking beschreibt den Prozess der Schaffung eines gemeinsam konstruierten und koordinierten Systems des Handelns in Organisationen. In diesem Prozess manifestiert sich eine gemeinsame Haltung, welche dazu dient, Identität und Aktion in organisationalem Handeln zu definieren (Weick et al., 2005, S. 409). In der schulischen Alltagspraxis könnte man sich darunter beispielsweise ein Leitbild oder ein Konzeptpapier vorstellen, wobei dies nicht zwingend niedergeschrieben sein muss, sondern auch nicht kommuniziertes Organisationswissen beinhalten kann. Prozesse des Sensemakings werden als aktive Aushandlung unter Bezugnahme auf retrospektive Erfahrungen beschrieben. Durchlaufen werden die Stadien der Differenzierung, Identifizierung, Klassifizierung, Regulierung und Routinierung (ebd., S. 411). Das Ziel des organisationalen Sensemakings kann darin gesehen werden, Plausibilität und Einigkeit im Umgang mit Problemen zu generieren. Dadurch wird eine überindividuelle geteilte Organisationsidentität erschaffen (Weick et al., 2005, S. 416-417). Organisationsidentitäten müssen nicht zwingend stabil gedacht werden. Giddens (1997) zufolge unterliegen sie vielmehr Aushandlungsprozessen durch Reflexion, Steuerung und Rationalisierung unterschiedlicher Interessen.

Ausgehend von diesen konzeptionellen Erwägungen können Schulen als besondere Organisationen beschrieben werden, die auf lose gekoppelten Zusammenhängen zwischen verschiedenen Akteuren basieren, welche sich zueinander und zu ihrem Kontext in ein Verhältnis setzen müssen. Konstitutiv sind somit Rekontextualisierungsleistungen auf verschiedenen Ebenen sowie Aushandlungsprozesse, ob und was gemeinsame Handlungsgrundlage in der Organisation sein soll. In einer organisationstheoretischen Perspektive kommt dabei die Rolle der Organisation als eigenständige Sinnstruktur unbewusster Handlungsbezüge ins Spiel. Da die Luhmann'sche Systemtheorie einen zentralen organisationstheoretischen Bezug darstellt, wird im nächsten Abschnitt auf ihrer Grundlage die Herausbildung organisationsinterner Handlungsroutinen und polykontexturaler Arrangements betrachtet.

\subsection{Sinnherstellung in Organisationen aus system- und organisationstheoretischer Sicht}

Wenn von Organisationen und deren Sinnherstellung gesprochen wird, kann als ein zentraler Bezugspunkt die Systemtheorie nach Luhmann (1984) ausgemacht werden. Systeme werden dabei nicht nur auf Gesellschaftsebene (Systemebene) festgemacht, sondern ebenfalls auf der Ebene von Organisationen (Luhmann, 2006). Luhmanns Sichtweise der Sinnherstellung in Organisationen ist für eine organisationssoziologi- 
sche Perspektive richtungsweisend und kann daher als eine Grundlage organisationstheoretischer Reflexionen angesehen werden. In Auseinandersetzung mit und Abgrenzung von der strukturfunktionalistischen Systemtheorie nach Parsons (1902-1979) entwickelte Luhmann (1984) eine soziologische Systemtheorie, welche auf der Differenz zwischen System und Umwelt aufbaut (zit. nach Luhmann \& Baecker, 2011). Diese Abgrenzung bezieht sich insbesondere auf die Annahme des Vorhandenseins gesellschaftlich gleichförmiger, internalisierter kultureller Wertsysteme. Neben immanenten Schwächen in Parsons' Ansatz, wie unter anderem der fehlenden Erklärung dysfunktionalen Verhaltens, kritisiert Luhmann (2011, S. 17) insbesondere die unterstellte Unveränderlichkeit von Systemen und fordert eine Entwicklungsperspektive.

An die strukturfunktionalistische Perspektive des Zusammenhangs von Struktur und Handeln anknüpfend, erweitert Luhmann den Ansatz zu einer neuen Theorie des Zusammenspiels sozialer Systeme (Luhmann, 1984). Soziale Systeme und Organisationen bestehen dabei aus Kommunikation, mit der sie sich rekursiv auf ihre Umwelt beziehen (Luhmann, 2006, S. 59). Durch Kommunikation werden Relationen und Differenzen zu anderen Systemelementen produziert, wodurch der Ansatz unterscheidungs- und differenztheoretisch geprägt ist. Organisationen zeichnen sich gemäß Luhmann (2006, S. 39-41) durch Autopoiesis und damit durch operative Geschlossenheit und Selbstreferenz in der Sinnherstellung, keineswegs jedoch durch Strukturkonservatismus aus. Eine Organisation als System grenzt sich von seiner Umwelt ab, befindet sich aber gleichzeitig in einem Dauerzustand der Unsicherheit und Sinnsuche (Luhmann, 2006, S. 47-48). Die Umwelt wird als Konstrukt oder Fremdreferenz der Organisation verstanden; sie hilft dabei, Entscheidungen des Systems retrospektiv nachzuvollziehen (ebd., S. 52).

Wird sie auf Organisationen des Erziehungssystems bezogen, erkennt die Theorie insbesondere die Selektion in Auseinandersetzung mit den pädagogischen Idealen der Akteure als zentrale Aufgabe der Schule. So stellen Luhmann \& Lenzen (2002) in Das Erziehungssystem der Gesellschaft Erziehung als pädagogisches Ideal und Selektion als staatlich aufgezwungene Anforderung einander gegenüber:

\begin{abstract}
„Aber selbst wenn man die Mindererfolgreichen mit besonderen Maßnahmen förderte, stellte sich das Problem auch in dieser Gruppe ein: die einen erweisen sich gleicher als die anderen; die einen schafften es, die anderen nicht. Wir müssen deshalb davon ausgehen, daß Selektion sich nicht vermeiden läßt, wenn Erziehung sich als gute Absicht vorstellt und das Richtige markiert. Auch die Schüler übernehmen die Ergebnisse der Selektionsverfahren als Qualitätszeugnisse in ihre wechselseitige Einschätzung “ (Luhmann \& Lenzen, 2002, S. 63).
\end{abstract}

Das Zitat macht die Auseinandersetzung zwischen der Fremd- und der Selbstreferenz von schulischen Organisationen deutlich. Selektionsmechanismen werden als nicht vermeidbarer Teil pädagogischen Handelns beschrieben. Eine zentrale Aufgabe von schulischen Organisationen ist es demnach, Leistungsunterschiede zu produzieren. 
Diese in das System eingeschriebene Funktion wird von Pädagogen und Schülerinnen bzw. Schülern übernommen und gelebt.

Dass individuelle Entscheidungen und Handlungsfreiheiten ausgeblendet werden, kann denn auch als Hauptkritik an dem Ansatz gesehen werden. Kommunikation erscheint aus dieser Sicht als Element sozialer Systeme, nicht als etwas, das zwischen Personen stattfindet; Akteure und deren Handeln werden mehrheitlich nicht berücksichtigt. Trotz der Kritik an der fehlenden Dynamik bzw. den fehlenden Weiterentwicklungsmöglichkeiten der Systeme im Strukturfunktionalismus, kann sich der theoretische Ansatz von Luhmann diesem Vorwurf ebenfalls nicht ganz entziehen. Die Abgrenzung von der Umwelt als zentrale Aufgabe von Organisationen führt gemäß Luhmann (2006, S. 55) zur Erzeugung von Selbstreferenz. Veränderungen der Selbstreferenz sind nur unter gewissen Voraussetzungen denkbar.

Luhmanns systemtheoretischer Ansatz kann trotz der genannten Kritikpunkte als Grundlage für die Reflexion der Sinnherstellung in Organisationen herangezogen werden. Selbstreferenzielle Systeme werden als Bezugspunkt von Handlungsorientierungen betrachtet. Der Ansatz der Polykontextur erlaubt es, in einer system- und organisationstheoretischen Perspektive die Überlagerung verschiedener handlungsleitender Kontexturen zu diskutieren. Das Konzept der Polykontexturalität geht davon aus, dass sich verschiedene sinngebende Strukturen in Organisationen überlagern (Vogd, 2009, S. 25). Marotzki verweist in seiner theoretischen Reflexion des Konzepts der Polykontexturalität darauf, dass es Kennzeichen von Bildungsprozessen ist, „Bestimmtheit (im Sinne der Strukturstabilität) herzustellen und Unbestimmtheit (im Sinne der Strukturtransformation) zu ermöglichen" (Marotzki, 1990, S. 118). Kontexturen werden als kommunikative, überindividuelle Reflexionsverhältnisse verstanden, wobei man bei einer Überlagerung unterschiedlicher Kontexturen von „,polykontexturalen Arrangements“" spricht (Vogd \& Jansen, 2013). Das Konzept lässt sich zur Erklärung von Entscheidungsfindungsprozessen in Organisationen verwenden (ebd.). Die soziale Praxis in Organisationen wird dabei als Resultat der Aushandlung unterschiedlicher bewusster und unbewusster Wissensbestände verstanden (Jansen, Schlippe \& Vogd, 2015). Es wird das Bestehen einer „Vielheit von Rationalitäten“ in Organisationen angenommen, die das Handeln anleiten (Vogd \& Jansen, 2013, S. 17).

Im Anschluss an das Konzept der Polykontexturalität nach Vogd und Jansen sowie in einer systemischen Perspektive auf Organisationen reflektieren Hartung-Beck und Muslic (2015) unterschiedliche Subsysteme innerhalb der Organisation. Solche Subsysteme - beispielsweise Schulleitung, Lehrkräfte, Schüler/-innen - können als in sich geschlossene Funktionssysteme angesehen werden, welche sich jedoch in einem wechselseitigen Abhängigkeitsverhältnis befinden (Hartung-Beck \& Muslic, 2015). Als polykontexturale Arrangements werden die strukturellen Koppelungen zwischen den Subsystemen zur Bewältigung der Wahl verschiedener Entscheidungsalternativen verstanden (Hartung-Beck \& Muslic, 2015). Polykontexturen finden sich in dieser Sicht auf einer kollektiven Ebene schulischer Funktionsgruppen. Funktionsgruppen 
meint in einer organisationssoziologischen Logik dabei nicht nur Professions- und Klientelgruppen, sondern auch funktionelle Zusammenschlüsse verschiedener schulischer Akteure mit einer gemeinsamen Zielsetzung. Beispielsweise sind Zürcher Volksschulen dazu aufgefordert, sich in Steuergruppen (interdisziplinär und stufenübergreifend), pädagogischen Teams (interdisziplinärer Austausch auf einer Stufe) sowie im Fall von QUIMS-Schulen zudem in QUIMS-Teams zu organisieren (Kanton Zürich Volksschulamt, 2008). Solche Akteursgruppen können ebenfalls als Subsysteme in Organisationen mit eigener Rationalität verstanden werden.

Die Forschung zu schulischen Funktionsgruppen bezieht sich insbesondere auf die Wirkung von Steuergruppen und der Schulleitung in Entwicklungsprozessen. Beispielsweise zeigt Feldhoff in einer quantitativen Evaluationsstudie zur Begleitung des Modells „Selbständige Schule“, dass Steuergruppen neben der Schulleitung als „Change Agent schulischer Entwicklungsprozesse“ das organisationale Lernen befördern können (Feldhoff, 2011, S. 284). Dabei wird dahingehend relativiert, dass die untersuchten Steuergruppen diese Rolle noch zu wenig wahrnehmen (Feldhoff, 2011). Eine mögliche Erklärung für das Ausbleiben einer dominierenden Rolle der Steuergruppe liefert die qualitativ-rekonstruktive Studie von Schröck und Rahm (2009). Die Autoren beschreiben schulische Steuergruppen in einem Spannungsverhältnis zwischen organisationalen und professionalen Anforderungen, wobei ihnen die Aufgabe zukommt, die lose gekoppelten Systeme zu verbinden (Schröck \& Rahm, 2009). Aus den Gruppendiskussionen mit fünf Steuergruppen geht hervor, dass der Prozess der Rollenfindung von der ständigen Positionierung zwischen Schulleitung und Kollegium geprägt ist (Schröck \& Rahm, 2009, S. 164). Dabei sind auch schulspezifische Unterschiede in den Hierarchiestrukturen sichtbar, nach denen sich die Steuergruppen richten (Schröck \& Rahm, 2009). Diese Studien geben Hinweise darauf, dass Steuergruppen als Beispiel schulischer Funktionsgruppen eine eigene schulische Kontextur darstellen, welche sich durch die organisationsspezifische Einbettung sowie innerorganisatorische Aushandlungsprozesse definiert.

Insgesamt erlaubt die systemtheoretische Betrachtungsweise es, die schulische Organisation als eine sinngebende Struktur anzusehen. Zugleich werden weitere Handlungsbezüge in die Diskussion einbezogen. Geht man der Frage nach, welche Akteure oder Kollektive an der Herausbildung geteilter Sinnmuster beteiligt sind, liefert das Konzept der Polykontextur Hinweise. Innerorganisationale Subsysteme mit eigenen Rationalitäten stehen bei der kollektiven Sinnsuche in einem Aushandlungsprozess mit außerorganisatorischen Bezügen. Zu fragen ist, ob dabei ein gemeinsames überindividuelles Wertsystem entsteht. Das Konzept der Schulkultur kann dabei helfen, den Sinnsuchprozess in schulischen Organisationen weiter theoretisch zu reflektieren. 


\subsection{Schulkultur}

Mögliche Hinweise für ein überindividuell geteiltes Wertsystem als Endprodukt innerorganisationaler Sinnsuchprozesse liefert der Schulkulturansatz. Ähnlich wie bei Fend (2008) und dem Konzept der Rekontextualisierung wird von einer relativierten Außensteuerung ausgegangen und nach dem Eigensinn pädagogischer Organisationen gefragt (Helsper, 2008). Der Schulkulturansatz wurde von Werner Helsper seit Mitte der 1990er Jahre basierend auf einem rekonstruktiven Projekt zu Transformationsprozessen entwickelt und im Anschluss von ihm und anderen weiter ausgearbeitet (vgl. dazu Kramer, 2015).

Helspers Schulkulturansatz integriert sowohl system- und strukturtheoretische als auch akteurstheoretische Perspektiven. Zudem lassen sich anerkennungstheoretische Bezüge ausmachen (vgl. Kramer, 2015, S. 32). Der Schulkulturansatz wird von Helsper und Kollegen (2001) am Beispiel von hermeneutisch-rekonstruktiven Fallstudien deutscher Gymnasien empirisch auf grundlegende Strukturproblematiken und deren interaktive Bearbeitungen auf Schulebene geprüft. Dabei kommen die Autoren zu dem Schluss, dass schulische Akteure in der Auseinandersetzung mit ihren sozialen Kontextbedingungen, Strukturproblemen, Strukturvorgaben und pädagogischen Antinomien einzelschulspezifische symbolische Sinnstrukturen entwickeln, reproduzieren und transformieren (Helsper, Böhme, Kramer \& Lingkost, 2001, S. 535). In der Kritik an der Output-orientierten Forschung ,guter Schulen“ (wie u. a. Rolff, 1993) betonen die Autoren die Prozesshaftigkeit schulischer Kulturen, die das Ergebnis von Aushandlungsprozessen zwischen sozialen, organisationalen und professionellen Sinnstrukturen sind (Helsper et al., 2001, S. 536-537). Dabei wird untersucht, inwiefern eine dominante Schulkultur als Möglichkeitsraum und Begrenzung des professionellen Handelns in Schulen wirkt. Helsper et al. (2001, S. 26) verstehen Schulkultur nicht einfach als pädagogischen oder organisationalen Konsens, sondern vielmehr als Aushandlungsprozess verschiedener Schulakteure in der Ausgestaltung einer ,symbolischen Ordnung" der Einzelschule. Hinsichtlich des Kulturaspekts bezieht der Ansatz auf der Ebene der Tiefenstruktur anerkennungstheoretische Reflexionen unter Bezugnahme auf Honneth ein (ebd., S. 31). Anerkennungsverhältnisse werden dabei zwischen verschiedenen Schulakteuren in der Aushandlung einer dominanten symbolischen Ordnung, zentraler noch als pädagogische Prämisse der Beziehung zwischen Lehrpersonen und Schülerinnen bzw. Schülern reflektiert (ebd., S. 31). Der Schulkulturansatz kann daher als eine mehrperspektivische Reflexion auf die Frage kollektiver, pädagogisch gehaltvoller Sinnordnungen in Organisationen angesehen werden. Helsper (2008) definiert Schulkultur mit Blick auf ihre Bedeutung für die pädagogische Professionalität folgendermaßen:

„Schulkulturen sind als die symbolische Ordnung der einzelnen Schulen in der Spannung von Realem, Symbolischem und Imaginärem zu fassen. (...) Die jeweilige Schulkultur stellt die einzelschulspezifische Strukturvariante dar, in der die Strukturprobleme 
des Bildungssystems und die grundlegende Antinomen des pädagogischen Handelns die selbst sinnkonstituiert sind - je spezifisch gedeutet werden und in symbolischen pädagogischen Formen, Artefakten, Praktiken, Regeln, imaginären pädagogischen Sinnentwürfen und schulischen Mythen ihren jeweiligen Ausdruck finden" (Helsper, 2008, S. 122).

Die symbolische Ordnung der Einzelschule wird somit als Aushandlungsprozess verstanden. Solche Aushandlungsprozesse sind eingebettet in schulspezifische Strukturen und Vorgaben. Hinsichtlich der Bedeutung für die pädagogische Profession wird in der Definition aber auch eine akteursperspektivische Sicht auf den pädagogischen Habitus und die berufsbiographischen Passungsverhältnisse einbezogen. In der Spannung zwischen imaginären programmatischen Zielsetzungen, dem Bezug zu realen Kontextbedingungen und der symbolisch interaktiven Bearbeitung zwischen Zielen und der Realität auf Organisationsebene als Grundlage für die Bearbeitung spezifischer Strukturprobleme gestalten sich verschiedene Strukturvarianten einer Schulkultur (Helsper, 2008). Dabei knüpft Helsper an die Theorie der Strukturierung nach Giddens (1997) an, um sowohl akteurs- als auch systemperspektivische Muster in den Strukturprinzipien der Institutionen zu erklären, auf welche die Akteure ihr Handeln beziehen (zit. nach Kamm, 2007).

In der Weiterentwicklung des Konzepts bezieht sich Helsper (2008) insbesondere auf das dialektische Modell der Strukturierung von Sozialität nach Oevermann. Dieses stellt äußeren Regeln eine Fallstruktur oder Identität in der Handlungswahl von Individualität und Kollektivität gegenüber. Dabei kombiniert der Schulkulturansatz eine mikropolitische Sicht auf den berufs- und milieuspezifischen Habitus der Lehrperson in Aushandlung mit einer Schulkultur, die auf dominanten pädagogischen Sinnentwürfen im Einzelschulkontext basiert (Helsper, 2008). Die Herausbildung einer Schulkultur eröffnet eine organisationale Autonomie gegenüber bildungspolitischen Strukturen und soziokulturellen Bedingungen, indem ein gemeinsames pädagogisch-professionelles Handlungswissen geschaffen wird (Helsper, 2008). Die Schulkultur kann jedoch auch de-professionalisierend wirken, wenn sie in Widerspruch zu dem professionsspezifischen und biographischen Erfahrungswissen der Einzelakteure steht (vgl. Helsper, 2008, S. 135-137).

Der Ansatz der Schulkultur bietet eine kombinierte Betrachtung von strukturtheoretischen, organisationstheoretischen und mikropolitischen Perspektiven auf kollektive Sinnsuchprozesse in Organisationen. Schulkultur meint nicht nur ein formales Regelwerk, sondern bezieht sich in seiner Tiefenstruktur auf pädagogische Prämissen zwischen Schülerinnen bzw. Schülern und Lehrpersonen. Kultur wird transformierend gedacht, im ständigen Aushandlungsprozess zwischen hierarchisch strukturierenden Dominanzmustern, pädagogischen Sinnentwürfen und kontextualen Bezügen. Schulkultur kann als überindividueller und dynamischer Erklärungsansatz angesehen werden, wobei Kultur weniger als normativ erwünschtes Endergebnis, sondern vielmehr als Aushandlungsprozess verschiedener Akteure verstanden wird. 


\section{Fazit}

Bei der Frage, inwiefern die Organisation selbst, aber auch die verschiedenen Akteure und Akteursgruppen für geteilte oder divergierende Vorstellungen und Konzeptionen hinsichtlich Förderung, Selektion und Bildungsgerechtigkeit struktur- und sinngebend sind, gibt es unterschiedliche theoretische Ansätze. Verschiedene theoretische Bezüge und empirische Studien weisen darauf hin, dass auch nach überindividuellen Einflüssen auf pädagogische Konzeptionen zu fragen ist. Zielführend erscheint es daher, sowohl akteurszentrierte als auch überindividuelle und organisationale Ursachen für die Herstellung von Sinn in schulischen Organisationen zu betrachten und die entsprechenden theoretischen Konzepte kombiniert zugrunde zu legen. Kollektive oder auch divergierende pädagogische Konstruktionen sind in einer systemtheoretischen Sichtweise ein Ergebnis von Aushandlungsprozessen, an denen verschiedene pädagogische Akteure, Subsysteme und Systeme beteiligt sind. Die soziologische Systemtheorie von Luhmann, welche die Selbst- und Fremdreferenz sowie die Geschichtlichkeit gesellschaftlicher Subsysteme ins Zentrum stellt, kann als Basis für die Sinnherstellung in Organisationen angesehen werden. Auf die Frage, weshalb Rahmenbedingungen einzelschulisch unterschiedlich adaptiert werden, bietet der mehrperspektivische Zugang des Rekontextualisierungsansatzes eine mögliche Antwort. Die Sinnherstellung von Organisationen als aktiver, retrospektiver Prozess wird weiter im Sensemaking-Ansatz von Weick thematisiert. Dabei wird davon ausgegangen, dass überindividuelles Handlungswissen in Organisationen existiert. Noch expliziter wird die Vielschichtigkeit pädagogischen Handelns im Konzept der Polykontextur aufgenommen, das von einer Vielheit von Rationalitäten ausgeht und dem Aushandlungsprozess verschiedener Kontexturen in der Sinnherstellung schulischer Organisation nachgehen möchte. Der Schulkulturansatz schließlich nimmt ergänzend eine Struktur- und Akteursperspektive ein, wobei in Erweiterung des Sensemaking-Konzepts nicht nur nach gemeinsamen Routinen, sondern zudem nach der Tiefenstruktur gefragt wird. Insofern geht es auch um die symbolischen Ordnungen an Schulen, wobei das pädagogische Handeln auf eine dominante Leitkultur ausgerichtet ist. Dieser pädagogische Aspekt wird in einer anerkennungstheoretischen Perspektive analysiert. 
Open Access Dieses Kapitel wird unter der Creative Commons Namensnennung 4.0 International Lizenz (http://creativecommons.org/licenses/by/4.0/deed.de) veröffentlicht, welche die Nutzung, Vervielfältigung, Bearbeitung, Verbreitung und Wiedergabe in jeglichem Medium und Format erlaubt, sofern Sie den/die ursprünglichen Autor(en) und die Quelle ordnungsgemäß nennen, einen Link zur Creative Commons Lizenz beifügen und angeben, ob Änderungen vorgenommen wurden. Die in diesem Kapitel enthaltenen Bilder und sonstiges Drittmaterial unterliegen ebenfalls der genannten Creative Commons Lizenz, sofern sich aus der Abbildungslegende nichts anderes ergibt. Sofern das betreffende Material nicht unter der genannten Creative Commons Lizenz steht und die betreffende Handlung nicht nach gesetzlichen Vorschriften erlaubt ist, ist für die oben aufgeführten Weiterverwendungen des Materials die Einwilligung des jeweiligen Rechteinhabers einzuholen.

(c) (1) 